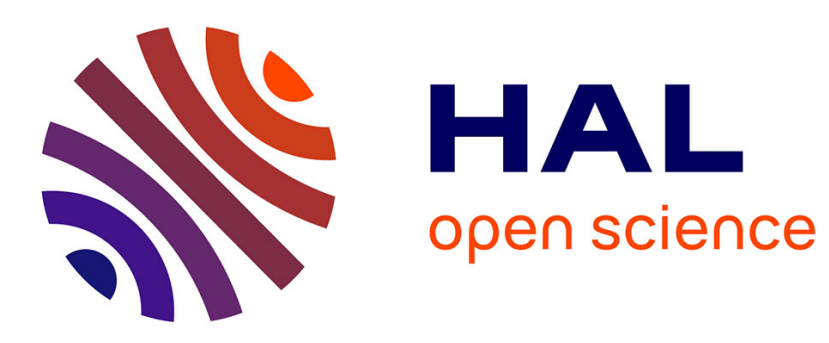

\title{
Generalized convex functions and generalized differentials
}

\author{
Thi Hong Linh Nguyen, Jean-Paul Penot
}

\section{To cite this version:}

Thi Hong Linh Nguyen, Jean-Paul Penot. Generalized convex functions and generalized differentials. 2006. hal-00423367

\section{HAL Id: hal-00423367 \\ https://hal.science/hal-00423367}

Preprint submitted on 9 Oct 2009

HAL is a multi-disciplinary open access archive for the deposit and dissemination of scientific research documents, whether they are published or not. The documents may come from teaching and research institutions in France or abroad, or from public or private research centers.
L'archive ouverte pluridisciplinaire HAL, est destinée au dépôt et à la diffusion de documents scientifiques de niveau recherche, publiés ou non, émanant des établissements d'enseignement et de recherche français ou étrangers, des laboratoires publics ou privés. 


\title{
Generalized convex functions and generalized differentials
}

\author{
Nguyen Thi Hong Linh and Jean-Paul Penot \\ Laboratoire de Mathématiques, CNRS UMR 5142, \\ Faculté des Sciences, Av. de l'Université 64000 Pau, France (honglinh98t1@yahoo.com) \\ Laboratoire de Mathématiques, CNRS UMR 5142, \\ Faculté des Sciences, Av. de l'Université 64000 Pau, France (jean-paul.penot@univ-pau.fr).
}

\begin{abstract}
We study some classes of generalized convex functions, using a generalized differential approach. By this we mean a set-valued mapping which stands either for a derivative, a subdifferential or a pseudodifferential in the sense of Jeyakumar and Luc. We establish some links between the corresponding classes of pseudoconvex, quasiconvex and another class of generalized convex functions we introduced. We devise some optimality conditions for constrained optimization problems. In particular, we get Lagrange-KuhnTucker multipliers for mathematical programming problems.

Key words: colinvex, generalized differential, mathematical programming, optimality conditions, protoconvex function, pseudoconvex function, quasiconvex function.
\end{abstract}

Mathematics Subject Classification: 26B25, 46G05, 49K27, 90C26, 90C32

\section{Introduction}

Various needs have led mathematicians to introduce and study several notions of generalized convexity or concavity. Among the tools used to define or study these notions are the various subdifferentials of nonsmooth analysis ([1], [17], [16], [24], [29], [32], [34], [39], [35]...), the convexificators of [11], the pseudo-differentials of Jeyakumar and Luc $([18])$, the normal cones to sublevel sets $([2],[4],[5],[6])$ and the generalized directional derivatives ([19], [20], [22], [40]). In the present paper we use a concept of generalized derivative which can encompass all these notions but the last one. We call it a generalized differential in contrast with the notion of subdifferential because it is not necessarily a one-sided concept. It allows much flexibility. It also leads us to get rid of some assumptions required in previous works such as smooth renorming of the space. We establish comparisons with the notions obtained using directional derivatives or their substitutes.

The case of pseudo-affine functions (also called pseudo-linear functions) is studied elsewhere ([23]) with similar concepts and methods. It requires some results of the present paper.

In the two last sections of the paper, we investigate some necessary and sufficient optimality conditions for constrained problems and mathematical programming problems.

\section{Characterizations of generalized convex functions}

We devote this preliminary section to review some concepts of generalized convexity; we also introduce new ones. Some elementary properties and characterizations are given.

In the sequel $X, Y$ are normed vector spaces, $X^{*}$ is the dual space of $X, C$ is a nonempty subset of $X$ and $f: C \rightarrow \mathbb{R}_{\infty}:=\mathbb{R} \cup\{+\infty\}$. We extend $f$ to $X \backslash C$ by $+\infty$; alternatively, we may consider $f: X \rightarrow \mathbb{R}_{\infty}$ and take for $C$ a subset of $X$ containing the domain of $f$. We denote by $\mathbb{P}$ (resp. $\mathbb{R}_{+}$) the set of positive numbers (resp. non negative numbers) and $\overline{\mathbb{R}}$ stands for $\mathbb{R} \cup\{-\infty,+\infty\}$. We assume that a set-valued map $\partial f: C \rightrightarrows X^{*}$ is given which stands as a substitute to the derivative of $f$; we call it a generalized differential of $f$. Among possible choices for $\partial f$ are the subdifferentials of $f$ in the various senses of nonsmooth analysis 
and the pseudo-differentials of $f$ in the sense of [18]. These cases will be considered below. We also deal with some other cases, such as normal cones set-valued maps.

We recall that the visibility cone $V(C, x)$ of $C$ at $x \in C$ is the cone generated by $C-x$ :

$$
V(C, x):=\mathbb{P}(C-x):=\{r(c-x): r \in \mathbb{P}, c \in C\}
$$

It contains the radial tangent cone to $C$ at $x$ which is the set

$$
T^{r}(C, x):=\left\{u \in X: \exists\left(r_{n}\right) \rightarrow 0_{+}, x+r_{n} u \in C \forall n\right\}
$$

The visibility bundle (resp. the radial tangent bundle) of $C$ is the set

$$
\begin{aligned}
& V C:=\{(x, u) \in C \times X: \exists r \in \mathbb{P}, w \in C, u=r(w-x)\}=\bigcup_{x \in C}\{x\} \times V(C, x) \\
& \text { (resp. } \quad T^{r} C:=\left\{(x, u) \in C \times X: \exists\left(r_{n}\right) \rightarrow 0_{+}, x+r_{n} u \in C \forall n\right\}=\bigcup_{x \in C}\{x\} \times T^{r}(C, x)
\end{aligned}
$$

If $C$ is starshaped at $x$ (in the sense that for all $w \in C$ and $t \in[0,1]$ one has $(1-t) w+t x \in C$ ) one has $V(C, x)=T^{r}(C, x)$. Thus, if $C$ is convex, $V C=T^{r} C$.

The upper and the lower radial derivatives (or upper and lower Dini derivatives) of $f$ at $x \in C$ in the direction $u \in T^{r}(C, x)$, are defined by

$$
\begin{aligned}
& D^{+} f(x, u)=\limsup _{t \rightarrow 0+, x+t u \in C} \frac{1}{t}[f(x+t u)-f(x)] ; \\
& D_{+} f(x, u)=\liminf _{t \rightarrow 0+, x+t u \in C} \frac{1}{t}[f(x+t u)-f(x)] .
\end{aligned}
$$

The upper and the lower radial subdifferentials are defined by

$$
\begin{array}{ll}
\partial^{r} f:=\left\{x^{*}:\left\langle x^{*}, u\right\rangle \leq D^{+} f(x, u)\right. & \left.\forall u \in T^{r}(C, x)\right\} \\
\partial_{r} f:=\left\{x^{*}:\left\langle x^{*}, u\right\rangle \leq D_{+} f(x, u)\right. & \left.\forall u \in T^{r}(C, x)\right\} .
\end{array}
$$

The definitions of $\partial f$-pseudoconvexity and $\partial f$-quasiconvexity we adopt here for a generalized subdifferential $\partial f$ of $f$ are similar to the ones used for a subdifferential by several authors; see [29], [34] and the references therein. We also introduce definitions of $\partial f$-protoconvexity and strict $\partial f$-pseudoconvexity of $f$ as natural variants of the two preceding concepts.

Definition 1 Let $f: C \rightarrow \mathbb{R}_{\infty}, x \in$ domf. A function $f$ is said to be

(i) $\partial f$-pseudoconvex at $x$ if for all $w \in C$ :

$$
f(w)<f(x) \Rightarrow \text { for all } x^{*} \in \partial f(x):\left\langle x^{*}, w-x\right\rangle<0 .
$$

(ii) $\partial f$-quasiconvex at $x$ if for all $w \in C$ :

$$
f(w)<f(x) \Rightarrow \text { for all } x^{*} \in \partial f(x):\left\langle x^{*}, w-x\right\rangle \leq 0 .
$$

(iii) $\partial f$-protoconvex at $x$ if for all $w \in C$ :

$$
f(w) \leq f(x) \Rightarrow \text { for all } x^{*} \in \partial f(x):\left\langle x^{*}, w-x\right\rangle \leq 0 .
$$

(iv) strictly $\partial f$-pseudoconvex at $x$ if for all $w \in C \backslash\{x\}$ :

$$
f(w) \leq f(x) \Rightarrow \text { for all } x^{*} \in \partial f(x):\left\langle x^{*}, w-x\right\rangle<0 .
$$


We add the word "eventually" when in the preceding implications "for all" is changed into "there exists". Thus, for instance, $f$ is eventually $\partial f$-pseudoconvex at $x$ if for all $w \in C$ satisfying $f(w)<f(x)$ there exists $x^{*} \in \partial f(x)$ such that $\left\langle x^{*}, w-x\right\rangle<0$.

Remark that the definition of $\partial f$-strict pseudoconvexity is irrealistic if $x$ is a non unique minimizer of $f$. Clearly

$$
\begin{aligned}
f \text { is strictly } \partial f \text {-pseudoconvex at } x & \Longrightarrow f \text { is } \partial f \text {-protoconvex and } \partial f \text {-pseudoconvex at } x, \\
f \text { is } \partial f \text {-pseudoconvex at } x & \Longrightarrow f \text { is } \partial f \text {-quasiconvex at } x, \\
f \text { is } \partial f \text {-protoconvex at } x & \Longrightarrow f \text { is } \partial f \text {-quasiconvex at } x .
\end{aligned}
$$

We say that $f$ is $\partial f$-pseudoconvex on $C$ (in short $\partial f$-pseudoconvex) if it is $\partial f$-pseudoconvex at every $x \in C$. We use a similar convention for the other concepts introduced above.

Let us observe that $\partial f$-quasiconvexity is different from full $\partial f$-quasiconvexity (in the terminology of [29]) in the sense (due to D. Aussel [1]) that $\operatorname{dom} f$ is convex and for any $w, x \in C, x^{*} \in \partial f(x)$ with $\left\langle x^{*}, w-x\right\rangle>0$, one has $f(w) \geq f(z)$ for any $z \in[x, w]$. Clearly, if $f$ is $\partial f$-quasiconvex and quasiconvex then $f$ is fully $\partial f$-quasiconvex while a fully $\partial f$-quasiconvex function is obviously $\partial f$-quasiconvex.

The following examples show that versatility is gained by taking for $\partial f$ a multimap which may differ from usual subdifferentials such as the Fenchel, the Fréchet or the Dini-Hadamard subdifferentials.

Example 1. Let $f: \mathbb{R} \rightarrow \mathbb{R}$ be given by $f(x)=2 x$ for $x \in[-1,1], f(x)=x+1$ for $x>1, f(x)=x-1$ for $x<-1$. We can take $\partial f(x)=\partial^{D} f(x)$ when $x \neq 1$ and $\partial f(1)=-\partial^{D}(-f)(1)$ (where $\partial^{D} f$ is the Dini-Hadamard subdifferential of $f)$. Observe that $\partial^{D} f(1)=\varnothing$.

Example 2. Let $f: X \rightarrow \mathbb{R}$ be such that for some generalized differential $\partial^{\sharp}$ one has $\partial^{\sharp}(-f)(x) \neq \varnothing$ for all $x \in X$. Then it may be convenient to take $\partial f:=-\partial^{\sharp}(-f)(x)$.

Example 3. Let $f: X \rightarrow \mathbb{R}$ be such that $f=f_{1}+f_{2}$. Then one may take $\partial f:=\partial^{\sharp} f_{1}+\partial^{\sharp} f_{2}$, where $\partial^{\sharp}$ is some generalized differential, althougth it may be different from $\partial^{\sharp}\left(f_{1}+f_{2}\right)$.

Example 4. Let $f: X \rightarrow \mathbb{R}$ be such that $f=g-h$ for some functions $g, h$. Then one may take $\partial f(x):=$ $\partial^{\sharp} g(x) \boxminus \partial^{\sharp} h(x)$, where $\partial^{\sharp}$ is some generalized differential, and for subsets $A, B$ of $X^{*}, A \boxminus B:=\left\{x^{*} \in X^{*}\right.$ : $\left.B+x^{*} \subset A\right\}$, althougth $\partial f$ may be different from $\partial^{\sharp}(g-h)$.

For some subdifferentials the preceding definitions may be automatically satisfied. This is the case if $\partial f$ is the Fenchel-Moreau subdifferential $\partial^{F M} f$ given by $\partial^{F M} f(x):=\left\{x^{*} \in X^{*}: f \geq x^{*}+f(x)-x^{*}(x)\right\}$. Let us consider some other classical subdifferentials. We recall that the original Greenberg-Pierskalla's subdifferential is defined by

$$
\partial^{G P} f(x)=\left\{x^{*} \in X^{*}:\left\langle x^{*}, w-x\right\rangle \geq 0 \Rightarrow f(w) \geq f(x)\right\} .
$$

The lower subdifferential, or Plastria subdifferential of $f$ at some point $x$ of its domain $\operatorname{dom} f:=\{x \in X$ : $f(x) \in \mathbb{R}\}$ is the set

$$
\partial^{<} f(x):=\left\{x^{*} \in X^{*}: \forall w \in S_{f}^{<}(x), f(w)-f(x) \geq\left\langle x^{*}, w-x\right\rangle\right\}
$$

where $S_{f}^{<}(x):=S_{f}^{<}(f(x)):=f^{-1}((-\infty, f(x)))$ is the strict sublevel set of $f$ at $x$. We also recall the following variant, called the infradifferential or Gutiérrez subdifferential:

$$
\partial^{\leq} f(x):=\left\{x^{*} \in X^{*}: \forall w \in S_{f}(x), f(w)-f(x) \geq\left\langle x^{*}, w-x\right\rangle\right\},
$$

where $S_{f}(x):=S_{f}(f(x)):=f^{-1}((-\infty, f(x)])$ is the sublevel set of $f$ at $x$. For such subdifferentials one has the following obvious statement.

Lemma 2 Let $f: X \rightarrow \mathbb{R}_{\infty}$ and $x \in \operatorname{domf}$. Then

(a) $f$ is $\partial^{G P} f$-pseudoconvex at $x$;

(b) $f$ is $\partial^{<} f$-pseudoconvex at $x$;

(c) $f$ is $\partial \leq f$-protoconvex and $\partial \leq f$-pseudoconvex at $x$. 
We denote by $N(C, x)$ the normal cone at $x \in X$ to a subset $C$ of $X$ given by

$$
N(C, x):=\left\{x^{*} \in X^{*}:\left\langle x^{*}, w-x\right\rangle \leq 0 \quad \forall w \in C\right\}
$$

even when $C$ is nonconvex; of course, such a cone is mainly of interest in the case $C$ is convex. Some normal cone operators can be associated to a function $f: X \rightarrow \mathbb{R}_{\infty}$ as follows: for $x \in \operatorname{dom} f$,

$$
\begin{gathered}
N_{f}(x):=N\left(S_{f}(x), x\right):=\left\{x^{*} \in X^{*}:\left\langle x^{*}, w-x\right\rangle \leq 0 \quad \forall w \in S_{f}(x)\right\}, \\
N_{f}^{<}(x):=N\left(S_{f}^{<}(x), x\right):=\left\{x^{*} \in X^{*}:\left\langle x^{*}, w-x\right\rangle \leq 0 \quad \forall w \in S_{f}^{<}(x)\right\} .
\end{gathered}
$$

(see for example [4], [6], [29] for the case $f$ is quasiconvex). Clearly, any function $f$ is $N_{f}$-protoconvex and $N_{f}^{<}$quasiconvex at any $x \in \operatorname{dom} f$. Moreover, for any function $f, \partial^{G P} f\left(\operatorname{resp} . N_{f}, N_{f}^{<}\right.$) is the greatest generalized differential $\partial f$ of $f$, such that $f$ is $\partial f$-pseudoconvex (resp. $\partial f$-protoconvex, $\partial f$-quasiconvex).

Recall that $f$ is said to be semi-strictly quasiconvex if it is quasiconvex and for all $w, x, y \in C, x \in(w, y)$ one has $f(x)<f(y)$ whenever $f(w)<f(y)$.

Lemma 3 Let $f: X \rightarrow \mathbb{R}_{\infty}$ be a quasiconvex function and let $x \in C$ such that $f(x)>\inf f(X)$. If there is no local minimizer of $f$ on $L_{f}(x):=f^{-1}(f(x))$, in particular $f$ is semi-strictly quasiconvex, then $N_{f}^{<}(x)=N_{f}(x)$.

Moreover, if $f$ is radially upper semicontinuous, then $f$ is $N_{f} \backslash\{0\}$-pseudoconvex at $x$.

This last assertion is given in [2, Proposition 3] and [5, Proposition 2.7]; we give a direct, simple proof for the convenience of the reader.

Proof. Since there is no local minimizer of $f$ on $L_{f}(x)$ (that occurs when $f$ is semi-strictly quasiconvex), $S_{f}^{<}(x)$ is dense in $S_{f}(x)$, hence $N_{f}^{<}(x)=N_{f}(x)$.

Then, for all $x^{*} \in N_{f}(x) \backslash\{0\}$ and $w \in S_{f}^{<}(x)$ we have $\left\langle x^{*}, w-x\right\rangle<0$ since if $\left\langle x^{*}, w-x\right\rangle=0$, taking $u \in X$ with $\left\langle x^{*}, u\right\rangle>0$ we would have $w+t u \in S_{f}^{<}(x)$ for $t>0$ small enough, and $\left\langle x^{*}, w+t u-x\right\rangle>0$, a contradiction. Thus $f$ is $N_{f} \backslash\{0\}$-pseudoconvex at $x$.

We say that $\partial f$ is a valuable generalized differential if for all $a \in X$ and $b \in X$ with $f(b)>f(a)$ there exist $c \in[a, b):=[a, b] \backslash\{b\}$ and sequences $\left(c_{n}\right),\left(c_{n}^{*}\right)$ such that $\left(c_{n}\right) \rightarrow_{f} c, c_{n}^{*} \in \partial f\left(c_{n}\right)$ for each $n$ and

$$
\left\langle c_{n}^{*}, d-c_{n}\right\rangle>0 \quad \text { for all } d \in b+\mathbb{R}_{+}(b-a) \text { and all } n \in \mathbb{N} .
$$

The following result shows that for a valuable subdifferential, quasiconvexity, $\partial f$-quasiconvexity and $\partial f$ protoconvexity coincide under a mild continuity assumption. The last assertion follows from [34].

Proposition 4 (a) If $f: X \rightarrow \mathbb{R}_{\infty}$ is quasiconvex and $\partial f \subseteq \partial^{r} f$, then $f$ is $\partial f$-protoconvex hence $\partial f$ quasiconvex.

(b) Let $f: X \rightarrow \mathbb{R}_{\infty}$ be a radially continuous l.s.c. function and let $\partial f$ be a valuable generalized differential. If $f$ is $\partial f$-quasiconvex, then it is quasiconvex.

Proof. (a) Let $w, x \in C$ and $x^{*} \in \partial f(x)$ be such that $\left\langle x^{*}, w-x\right\rangle>0$. Then there exists $\left(t_{n}\right) \rightarrow 0_{+}$such that $f\left(x+t_{n}(w-x)\right)>f(x)$. Since $f$ is quasiconvex, one has $f(w)>f(x)$.

(b) It follows from [34] with a similar proof.

The following proposition is well known when $\partial f$ is the derivative of $f$ or a subdifferential of $f$.

Proposition 5 Let $C$ be open, let $f: C \rightarrow \mathbb{R}$ be radially upper semicontinuous and $0 \notin \partial f(x)$.

(a) If $f$ is $\partial f$-quasiconvex at $x$ then it is $\partial f$-pseudoconvex at $x$.

(b) If $f$ is eventually $\partial f$-quasiconvex at $x$ then it is eventually $\partial f$-pseudoconvex at $x$.

Proof. Let $w, x \in C$ be such that $f(w)<f(x)$.

(a) If $f$ is $\partial f$-quasiconvex at $x$ then for all $x^{*} \in \partial f(x),\left\langle x^{*}, w-x\right\rangle \leq 0$. Suppose that there exists $x^{*} \in \partial f(x)$ such that $\left\langle x^{*}, w-x\right\rangle=0$. Since $x^{*} \neq 0$, we can find $u \in X$ such that $\left\langle x^{*}, u\right\rangle=1$. By radial upper semicontinuity of $f$, there exists $\varepsilon>0$ such that $y:=w+\varepsilon u \in C$ and $f(y)<f(x)$. Since $f$ is $\partial f$-quasiconvex,

$$
0 \geq\left\langle x^{*}, y-x\right\rangle=\left\langle x^{*}, w-x\right\rangle+\varepsilon\left\langle x^{*}, u\right\rangle=\varepsilon>0,
$$


a contradiction. Hence, one has $\left\langle x^{*}, w-x\right\rangle<0$ for all $x^{*} \in \partial f(x): f$ is $\partial f$-pseudoconvex at $x$.

(b) If $f$ is eventually $\partial f$-quasiconvex at $x$, there exists $x^{*} \in \partial f(x)$, such that $\left\langle x^{*}, w-x\right\rangle \leq 0$. With a similar proof to the one in (a), one gets $\left\langle x^{*}, w-x\right\rangle<0$.

A comparison between $\partial f$-pseudoconvexity, quasiconvexity (in the usual sense) and semi-strict quasiconvexity is given in the next proposition. Similar comparisons have been made in [17, Prop. 11.5], [30], [40], [34] in case $\partial f$ is contained in the dag subdifferential $\partial^{\dagger} f$ defined there. We use the conditions:

(C) if for some $w, x \in C, f$ is constant on $[w, x]$, then one has $\left\langle x^{*}, w-x\right\rangle \leq 0$ for some $x^{*} \in \partial f(x)$.

(M) if $x$ is a local minimizer of $f$, then $0 \in \partial f(x)$.

Condition (C) is satisfied when $\partial f(x) \cap \partial^{D} f(x) \neq \varnothing$, where $\partial^{D} f$ is the Dini-Hadamard subdifferential of $f$, in particular when $f$ is Gâteaux-differentiable at each point of $C$ and $f^{\prime}(x) \in \partial f(x)$. Condition (M) is a natural condition which is satisfied by all sensible generalizations of the derivative used for minimization problems.

Let us recall that a function $f$ is radially nonconstant if one cannot find any line segment on which $f$ is constant.

The first assertion of the following proposition can be given when $f$ is a radially continuous l.s.c. function and $\partial f$ is a valuable generalized differential since if $f$ is $\partial f$-pseudoconvex, then it is $\partial f$-quasiconvex and, by Proposition 4, it is quasiconvex; see also in [1, Theorem 4.1] for the case $X$ admits a smooth renorming. A comparison of assertions (b) and (c) with [1, Proposition 5.2] is made in the example below. The proof of the last assertion is similar to the one of [1, Proposition 5.2]; note that one does not need that $\partial$ be valuable.

Proposition 6 Let $C$ be convex, let $f: C \rightarrow \mathbb{R}_{\infty}$ be $\partial f$-pseudoconvex and $\partial f(x) \neq \varnothing$ for all $x \in C$. Then

(a) $f$ is quasiconvex.

(b) If $f$ satisfies condition $(C)$, then it is semi-strictly quasiconvex.

(c) If $f$ satisfies condition ( $M)$, then every local minimizer is a global minimizer. Moreover, if $f$ is radially continuous and l.s.c., then it is semi-strictly quasiconvex.

(d) If $f$ is radially nonconstant, then $f$ is strictly $\partial f$-pseudoconvex.

Proof. (a) Let $f$ be $\partial f$-pseudoconvex. Suppose that $f$ is not quasiconvex. Then there exist $w, x, y \in C$ such that $x \in(w, y)$ and $f(x)>f(w), f(x)>f(y)$. By $\partial f$-pseudoconvexity of $f$, for all $x^{*} \in \partial f(x)$ one has $\left\langle x^{*}, w-x\right\rangle<0$ and $\left\langle x^{*}, y-x\right\rangle<0$, a contradiction with the linearity of $x^{*}$.

(b) Now, suppose that $f$ is $\partial f$-pseudoconvex and satisfies condition (C). If $f$ is not semi-strictly quasiconvex then there exist $w, x, y \in C$ with $x \in(w, y)$ such that $f(w)<f(y) \leq f(x)$. Thus, by (a), $f(x)=f(y)$ and $x$ is a maximizer of $f$ on $[w, x]$. Now, if $f([x, y])$ is not constant then there exists $z \in(x, y)$ such that $f(z)<f(x)$, a contradition with the quasiconvexity of $f$ and $f(w)<f(x)$. Hence, $f([x, y])$ is constant. Since $f$ is $\partial f$ pseudoconvex and $f(w)<f(x)$, one has $\left\langle x^{*}, w-x\right\rangle<0$ for all $x^{*} \in \partial f(x)$. On the other hand, by condition $(\mathrm{C})$, there exists $\bar{x}^{*} \in \partial f(x)$ such that $0 \geq\left\langle\bar{x}^{*}, y-x\right\rangle$ : a contradiction.

(c) The first assertion follows from the definition of $\partial f$-pseudoconvexity of $f$ and condition (M). For the second assertion, see [17, Prop. 11.5 (iii)]. Note that for this implication, one does not need that $\partial$ be valuable.

(d) See [1, Proposition 5.2].

Example 5. Let $f: \mathbb{R} \rightarrow \mathbb{R}$ be given by $f(x)=0$ for $x \leq 0$ and $f(x)=x$ for $x>0$. Since $f$ is not radially nonconstant, we cannot use [1, Proposition 5.2]. However, appropriate choices of $\partial f$ allow to apply Proposition 6 :

(a) Let $\partial f(x)=\mathbb{R}$ for $x<0$ and $\partial f(x)=\mathbb{P}$ for $x \geq 0$. Then $f$ is $\partial f$-pseudoconvex and condition (C) is satisfied. Thus, $f$ is semi-strictly quasiconvex. Note that condition $(\mathrm{M})$ is not satisfied since 0 is a local minimizer of $f$ but $0 \notin \partial f(0)$.

(b) If $\partial f(x)=\mathbb{R}$ for $x \leq 0$ and $\partial f(x)=\mathbb{P}$ for $x>0$, then condition (M) is satisfied.

A simple stability property is given in the following lemma (here the convexity of $C$ is not needed).

Lemma 7 Let $I$ be a finite set. For $i \in I$, let $f_{i}: C \rightarrow \mathbb{R}_{\infty}$, let $\partial f_{i}: C \rightrightarrows X^{*}$ and let $f:=\sup _{i \in I} f_{i}$. For

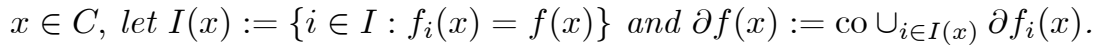

If for $i \in I(x), f_{i}$ is $\partial f_{i}$-pseudoconvex (resp. $\partial f_{i}$-protoconvex, $\partial f_{i}$-quasiconvex) at $x$, then $f$ is $\partial f$ pseudoconvex (resp. $\partial f$-protoconvex, $\partial f$-quasiconvex) at $x$. 
Proof. Let $w \in C$ be such that $f(w)<f(x)$. Then for all $i \in I(x)$ one has $f_{i}(w)<f_{i}(x)$. Since $f_{i}$ is $\partial f_{i}$-pseudoconvex, for all $x^{*} \in \cup_{i \in I(x)} \partial f_{i}(x)$, one has $\left\langle x^{*}, w-x\right\rangle<0$. Now, let $x^{*} \in \partial f(x)$. Then there exist $t_{i} \in[0,1], \sum_{i \in I(x)} t_{i}=1$ and $x_{i}^{*} \in \partial f_{i}(x)$ for $i \in I(x)$ such that $x^{*}=\sum_{i \in I(x)} t_{i} x_{i}^{*}$, hence $\left\langle x^{*}, w-x\right\rangle<0$.

A similar proof can be given to show that $f$ is $\partial f$-protoconvex or $\partial f$-quasiconvex at $x$.

\section{$3 \quad$ Links with previous works}

In [22], assuming a generalized directional derivative $h: V C \rightarrow \overline{\mathbb{R}}$ of $f$ is given, we defined concepts which seem to be related to the present notions. Let us make a precise comparison. Recall that $f$ is $h$-pseudoconvex (resp. h-quasiconvex, $h$-protoconvex) at $x$ if for all $w \in C$

$$
\begin{aligned}
f(w)<f(x) & \Rightarrow h(x, w-x)<0 \\
\text { (resp. } f(w)<f(x) & \Rightarrow h(x, w-x) \leq 0, \\
\text { resp. } f(w) \leq f(x) & \Rightarrow h(x, w-x) \leq 0) .
\end{aligned}
$$

Let us first consider the case we dispose of a general differential $\partial f$ which is a generalized pseudo-differential of $f$. The concept of pseudo-differential of $f$ has been introduced by Jeyakumar and Luc in [18] when $h^{+}$and $h_{+}$are the Dini derivatives of $f$. We introduce a slight variant which enables to encompass [14, Definitions $2.1,2.2]$ and [18].

Definition 8 Let $f: C \rightarrow \overline{\mathbb{R}}$ and $h: V C \rightarrow \overline{\mathbb{R}}$. We say that a subset $\partial f(x)$ of $X^{*}$ is:

(a) an upper h-pseudo-differential of $f$ at $x$ if for all $(x, u) \in V C$, we have

$$
h(x, u) \leq \sup \left\{\left\langle x^{*}, u\right\rangle: x^{*} \in \partial f(x)\right\} .
$$

(b) a lower h-pseudo-differential of $f$ at $x$ if for all $(x, u) \in V C$, we have

$$
h(x, u) \geq \inf \left\{\left\langle x^{*}, u\right\rangle: x^{*} \in \partial f(x)\right\} .
$$

(c) an $\left(h^{+}, h_{+}\right)$-pseudo-differential of $f$ at $x$, where $h^{+}, h_{+}: V C \rightarrow \overline{\mathbb{R}}$, if it is an upper $h^{+}$-pseudo differential of $f$ at $x$ and a lower $h_{+}$-pseudo differential of $f$ at $x$.

We say that $\partial f(x)$ is upper exact (resp. lower exact) if for all $u \in X$ one has

$$
\sup \left\{\left\langle x^{*}, u\right\rangle: x^{*} \in \partial f(x)\right\}=\max \left\{\left\langle x^{*}, u\right\rangle: x^{*} \in \partial f(x)\right\}
$$

(resp. $\left.\inf \left\{\left\langle x^{*}, u\right\rangle: x^{*} \in \partial f(x)\right\}=\min \left\{\left\langle x^{*}, u\right\rangle: x^{*} \in \partial f(x)\right\}\right)$ whenever the supremum (resp. infimum) is finite. Clearly, if $\partial f(x)$ is weak* compact then $\partial f(x)$ is upper exact and lower exact.

Proposition 9 Let $f: C \rightarrow \mathbb{R}_{\infty}, h: V C \rightarrow \overline{\mathbb{R}}$ and let $\partial f(x)$ be an upper h-pseudo-differential of $f$ at $x$. If $f$ is $\partial f$-quasiconvex (resp. $\partial f$-protoconvex) at $x$, then $f$ is h-quasiconvex (resp. $h$-protoconvex) at $x$.

If $f$ is $\partial f$-pseudoconvex at $x$ and if $\partial f(x)$ is upper exact, then $f$ is h-pseudoconvex at $x$.

Proof. Let $f$ be $\partial f$-quasiconvex (resp. $\partial f$-protoconvex) at $x$ and let $w \in C$ be such that $f(w)<f(x)$ (resp. $f(w) \leq f(x))$. Since $h(x, w-x) \leq \sup \left\{\left\langle x^{*}, w-x\right\rangle: x^{*} \in \partial f(x)\right\} \leq 0, f$ is $h$-quasiconvex (resp. $h$-protoconvex) at $x$.

Now let $f$ be $\partial f$-pseudoconvex at $x$ and let $w \in[f<f(x)]$. Then for all $x^{*} \in \partial f(x)$, one has $\left\langle x^{*}, w-x\right\rangle<0$. Thus, if $\partial f(x)$ upper exact, one has $\max \left\{\left\langle x^{*}, u\right\rangle: x^{*} \in \partial f(x)\right\}<0$, hence $h(x, w-x)<0$ and so $f$ is $h$ pseudoconvex at $x$.

We have a kind of converse.

Proposition 10 Let $\partial f(x)$ be a lower h-pseudo-differential of $f$ at $x$. If $f$ is h-pseudoconvex then $f$ is eventually $\partial f$-pseudoconvex.

If $f$ is h-quasiconvex (resp. h-protoconvex) at $x$ and if $\partial f(x)$ is lower exact, then $f$ is eventually $\partial f$ quasiconvex (resp. eventually $\partial f$-protoconvex) at $x$. 
Proof. Since $f$ is $h$-pseudoconvex at $x$, for any $w \in C, f(w)<f(x)$, one has

$$
0>h(x, w-x) \geq \inf \left\{\left\langle x^{*}, w-x\right\rangle: x^{*} \in \partial f(x)\right\} .
$$

Hence, there exists $x^{*} \in \partial f(x)$ such that $\left\langle x^{*}, w-x\right\rangle<0: f$ is eventually $\partial f$-pseudoconvex at $x$.

Now, let $f$ be $h$-quasiconvex (resp. $h$-protoconvex) at $x$. For any $w \in C, w \in[f<f(x)]$ (resp. $w \in[f \leq$ $f(x)])$, one has

$$
0 \geq h(x, w-x) \geq \inf \left\{\left\langle x^{*}, w-x\right\rangle: x^{*} \in \partial f(x)\right\} .
$$

Since $\partial f(x)$ is lower exact, there exists $x^{*} \in \partial f(x)$ such that $\left\langle x^{*}, w-x\right\rangle \leq 0$. Hence, $f$ is eventually $\partial f$ quasiconvex (resp. eventually $\partial f$-protoconvex) at $x$.

Now, let us assume that $\partial f$ is the generalized differential of $f$ associated with a bifunction $h$ in the following way:

$$
\partial f(x):=\partial^{h} f(x):=\partial h(x, \cdot)(0):=\left\{x^{*} \in X^{*}: \forall u \in X,\left\langle x^{*}, u\right\rangle \leq h(x, u)\right\} .
$$

We will use the following conditions on $h$ :

$$
\begin{array}{ll}
\forall w, x \in C & h(x, w-x)=\sup \left\{\left\langle x^{*}, w-x\right\rangle: x^{*} \in \partial^{h} f(x)\right\} . \\
\forall w, x \in C & h(x, w-x)=\max \left\{\left\langle x^{*}, w-x\right\rangle: x^{*} \in \partial^{h} f(x)\right\} .
\end{array}
$$

Condition (S) (resp. ( $\left.\left.\mathrm{S}^{\prime}\right)\right)$ is satisfied when $C$ is open and for all $x \in C$ the function $h(x, \cdot)$ is sublinear and l.s.c. (resp. continuous).

Proposition 11 (a) If $f$ is h-pseudoconvex (resp. h-quasiconvex, h-protoconvex) at $x$, then $f$ is $\partial^{h} f$-pseudoconvex (resp. $\partial^{h} f$-quasiconvex, $\partial^{h} f$-protoconvex) at $x$.

(b) Conversely, if $f$ is $\partial^{h} f$-pseudoconvex at $x$ and if $h$ satisfies condition ( $\left.S^{\prime}\right)$, then $f$ is h-pseudoconvex at $x$.

(c) If $f$ is $\partial^{h} f$-quasiconvex (resp. $\partial^{h} f$-protoconvex) at $x$ and if $h$ satisfies condition $(S)$, then $f$ is $h$ quasiconvex (resp. h-protoconvex) at $x$.

Proof. (a) If $f$ is $h$-pseudoconvex at $x$, then for $w \in[f<f(x)]$ and $x^{*} \in \partial^{h} f(x)$ one has $\left\langle x^{*}, w-x\right\rangle \leq$ $h(x, w-x)<0$. Thus $f$ is $\partial^{h} f$-pseudoconvex. Now, if $f$ is $h$-quasiconvex (resp. $h$-protoconvex) at $x$ then for $w \in[f<f(x)]$ (resp. $w \in[f \leq f(x)]$ ), $x^{*} \in \partial^{h} f(x)$, one has $\left\langle x^{*}, w-x\right\rangle \leq h(x, w-x) \leq 0$ and then $f$ is $\partial^{h} f$-quasiconvex (resp. $\partial^{h} f$-protoconvex) at $x$.

(b) It follows from Proposition 9, since when $h$ satisfies condition (S'), $\partial^{h} f(x)$ is an upper $h$-pseudodifferential of $f$ at $x$ which is upper exact.

(c) Since $h$ satisfy condition (S), $\partial^{h} f$ is an upper $h$-pseudo-differential of $f$ at $x$. Thus, the conclusion follows from the first assertion of Proposition 9.

\section{Optimality conditions for problems with constraints}

In the present section, we apply the preceding concepts to the constrained minimization problem

$(\mathcal{C}) \quad \min f(x)$ subject to $x \in C$,

where $C$ is a subset of $X$, and $f: C \rightarrow \mathbb{R}_{\infty}$. Let $S$ be the set of solutions to $(\mathcal{C})$ and let $\partial f: C \rightrightarrows X^{*}$ be a generalized differential of $f$.

Let us give a sufficient condition for $(\mathcal{C})$.

Proposition 12 (a) Let $f$ be $\partial f$-pseudoconvex at $a \in C$. If $0 \in \partial f(a)+N(C, a)$, then a is a solution to $(\mathcal{C})$.

(b) Let $f$ be eventually $\partial f$-pseudoconvex at $a \in C$. If $-\partial f(a) \subset N(C, a)$, then a is a solution to $(\mathcal{C})$.

(c) If $f$ is $\partial f$-pseudoconvex at $a$, if $\partial f(a)$ is upper exact and if the following condition holds, then $a$ is a solution to $(\mathcal{C})$ :

$$
\sup _{a^{*} \in \partial f(a)}\left\langle a^{*}, u\right\rangle \geq 0 \quad \forall u \in V(C, a) .
$$


Proof. (a) Let us note that the assumption of (a) is stronger than the assumption of (c). However, we give a direct proof. If $0 \in \partial f(a)+N(C, a)$, then there exists $a^{*} \in \partial f(a)$ such that $\left\langle a^{*}, x-a\right\rangle \geq 0$ for all $x \in C$. Since $f$ is $\partial f$-pseudoconvex at $a$, one has $f(x) \geq f(a)$ for all $x \in C: a \in S$.

(b) Suppose $-\partial f(a) \subset N(C, a)$ and $f$ is eventually $\partial f$-pseudoconvex at $a$. If for some $x \in C$ one has $f(x)<f(a)$ then there exists $a^{*} \in \partial f(a)$ such that $\left\langle a^{*}, x-a\right\rangle<0$, a contradiction with $-a^{*} \in N(C, a)$.

(c) Supposing that $a \notin S$ we show that (10) does not hold. Let $x \in C$ be such that $f(x)<f(a)$. Since $f$ is $\partial f$-pseudoconvex at $a$, for all $a^{*} \in \partial f(a)$, we have $\left\langle a^{*}, x-a\right\rangle<0$. Since $\partial f(a)$ is upper exact, we get $\max \left\{\left\langle a^{*}, x-a\right\rangle: a^{*} \in \partial f(a)<0\right.$ and $(10)$ does not hold.

It follows from Lemma 2 that the preceding proposition implies the result in [21, Prop. 5].

The following example shows that with some suitable choices of $\partial f$, the assumptions of the preceding proposition reduce to the condition $0 \in \partial f(a)+N(C, a)$.

Example 6. Let us take for $\partial f$ the subdifferentials $\partial^{0} f$ and $\partial^{\wedge} f$ given in [30, Example 7.2] as follows

$$
\begin{aligned}
& x^{*} \in \partial^{0} f(x) \Leftrightarrow f(x)=\min _{\left\langle x^{*}, w\right\rangle>1} f(w),\left\langle x^{*}, x\right\rangle>1, \\
& x^{*} \in \partial^{\wedge} f(x) \Leftrightarrow f(x)=\min _{\left\langle x^{*}, w\right\rangle \geq 1} f(w),\left\langle x^{*}, x\right\rangle \geq 1 .
\end{aligned}
$$

Then, any function $f$ is $\partial f$-pseudoconvex: if $x^{*} \in \partial^{0} f(x)$ is such that $\left\langle x^{*}, w-x\right\rangle \geq 0$, then $\left\langle x^{*}, w\right\rangle \geq\left\langle x^{*}, x\right\rangle>1$, hence $f(w) \geq f(x)$. Similarly, we have that $f$ is $\partial^{\wedge} f$-pseudoconvex.

The next proposition is similar to [4, Prop. 4.1]. Note that here, we do not need the quasiconvexity of $f$ and moreover $\partial f(x)$ may be different from $N_{f}^{<}(x) \backslash\{0\}$. Also, we do not need the additional assumptions of Proposition 5 which would enable to apply the preceding proposition.

Proposition 13 Let $C$ be convex and let $f$ be $\partial f$-quasiconvex and radially upper semicontinuous at a $\in C$. Assume that $0 \notin \partial f(a)$ and $C^{\perp}:=\left\{x^{*} \in X^{*}: \forall w, x \in C,\left\langle x^{*}, x\right\rangle=\left\langle x^{*}, w\right\rangle\right\}=\{0\}$. If $0 \in \partial f(a)+N(C, a)$, then $a$ is a solution to $(\mathcal{C})$.

Proof. Since $0 \in \partial f(a)+N(C, a)$, there exists $a^{*} \in \partial f(a)$ such that $\left\langle a^{*}, x-a\right\rangle \geq 0$ for all $x \in C$. Since $C^{\perp}=0$, there exists $w \in C$ such that $\left\langle a^{*}, w-a\right\rangle>0$. Now, for any given $x \in C, t \in(0,1)$, we define $x_{t}:=(1-t) x+t w$ so that

$$
\left\langle a^{*}, x_{t}-a\right\rangle=(1-t)\left\langle a^{*}, x-a\right\rangle+t\left\langle a^{*}, w-a\right\rangle>0
$$

Since $f$ is $\partial f$-quasiconvex at $a$, one has $f\left(x_{t}\right) \geq f(a)$ and then $f(x) \geq f(a)$ by radial upper semicontinuity of $f$ at $a$. Thus, $a \in S$.

When a suitable bifunction $h$ is available and $\partial f$ is an upper $h$-pseudo-differential of $f$, condition (10) turn to be necessary.

Proposition 14 Let $C$ be convex, $h: V C \rightarrow \mathbb{R}$ be such that $h(a, \cdot) \geq D_{+} f(a, \cdot)$ and let a subset $\partial f(a)$ of $X^{*}$ be an upper $h$-pseudo-differential of $f$ at a. If $a \in S$ then condition (10) holds.

Proof. Let $a \in S$ and $u \in V(C, a)$. Let $x \in C$ be such that $u:=x-a$. Then

$$
0 \leq D_{+} f(a, x-a) \leq h(a, x-a) \leq \sup \left\{\left\langle a^{*}, x-a\right\rangle a^{*} \in \partial f(a)\right\}
$$

Under some additional convexity assumptions, the sufficient condition of Proposition 12 (a) also is necessary.

Proposition 15 Let $C$ be convex, let $h: V C \rightarrow \mathbb{R}$ be such that $h(a, \cdot) \geq D_{+} f(a, \cdot)$ and let $\partial f(a)$ be an upper h-pseudo-differential of $f$ at a. Assume that $h(a, \cdot)$ is sublinear and continuous. If a $\in S$ then $0 \in$ $\mathrm{w}^{*}-\operatorname{cl}(\operatorname{co}(\partial f(a)))+N(C, a)$. In particular, if $\partial f(a)$ is weak* closed convex, one has $0 \in \partial f(a)+N(C, a)$. 
Note that the assumption that $\partial f(a)$ is weak* closed convex is satisfied when $\partial f(a)=\partial^{h} f(a)$.

Proof. Let $a \in S$. One has $h(a, v) \geq 0$ for all $v \in V(C, a)$ by [22, Proposition 27] or, equivalently, $h(a, \cdot)+\iota_{V(C, a)}(\cdot) \geq 0$. Since $C$ is convex, $V(C, a)$ is a convex set. Hence we have $0 \in \partial\left(h(a, \cdot)+\iota_{V(C, a)}(\cdot)\right)(0)$ so that $0 \in \partial h(a, \cdot)(0)+\partial \iota_{V(C, a)}(0)$. Now since $\partial f(a)$ is an upper $h$-pseudo-differential of $f$ at $a$, the set $\partial h(a, \cdot)(0)$ is contained in the weak* closed convex hull of $\partial f(a)$, which is $\partial f(a)$. Hence, we get $0 \in \partial f(a)+N(C, a)$.

Let us give some other optimality conditions in the spirit of Minty variational inequalities and [4]. Recall ([3]) that a multimap $T: C \rightrightarrows X^{*}$ is said to be upper sign-continuous on $C$ if, for every $w, x \in C$, the following implication (in which $x_{t}:=t x+(1-t) w$ ) holds:

$$
\forall t \in(0,1), \quad \inf \left\{\left\langle x_{t}^{*}, w-x\right\rangle: x_{t}^{*} \in T\left(x_{t}\right)\right\} \geq 0 \Rightarrow \sup \left\{\left\langle x^{*}, w-x\right\rangle: x^{*} \in T(x)\right\} \geq 0 .
$$

We will use the following assumption taken from ([3]):

(A) for every $x \in C$ there exists a convex neighborhood $V_{x}$ of $x$ and an upper sign-continuous operator $T: V_{x} \cap C \rightarrow 2^{X^{*}}$ with nonempty values satisfying $T(w) \subseteq \partial f(w)$ for all $w \in V_{x} \cap C$.

Proposition 16 Let $C$ be convex, let $\partial f: C \rightrightarrows X^{*}$ and let $f$ be $\partial f$-protoconvex on $C$. If $a \in S$, then for any $x \in C$ one has

$$
\inf \left\{\left\langle x^{*}, x-a\right\rangle: x^{*} \in \partial f(x)\right\} \geq 0 .
$$

If assumption (A) holds then condition (10) is satisfied. If moreover $T(a)$ is convex, $w^{*}$-compact, then $0 \in$ $\partial f(a)+N(C, a)$.

Proof. Suppose that there exist $x \in C$ and $x^{*} \in \partial f(x)$ such that $\left\langle x^{*}, x-a\right\rangle<0$. Since $f$ is $\partial f$-protoconvex at $x$, one has $f(a)>f(x)$ : a contradition with $a \in S$. Thus, $\inf \left\{\left\langle x^{*}, x-a\right\rangle: x^{*} \in \partial f(x)\right\} \geq 0$ for all $x \in C$.

Since, for every $x \in C$, there exists a convex neighborhood $V_{x}$ of $x$ such that for any $w \in V_{x} \cap C$, $T(w) \subseteq \partial f(w)$, one has

$$
\inf _{w \in V_{x} \cap C} \inf \left\{\left\langle w^{*}, w-a\right\rangle: w^{*} \in T(w)\right\} \geq 0 .
$$

Since $T$ is upper sign-continuous and $V_{x} \cap C$ is a convex set, for $w \in V_{x} \cap C$ and $t \in(0,1)$, we have $w_{t}:=$ $(1-t) a+t w \in V_{x} \cap C$ and $\inf \left\{\left\langle w_{t}^{*}, w_{t}-a\right\rangle: w_{t}^{*} \in T\left(w_{t}\right)\right\} \geq 0$ hence $\inf \left\{\left\langle w_{t}^{*}, w-a\right\rangle: w_{t}^{*} \in T\left(w_{t}\right)\right\} \geq 0$. By upper sign-continuity of $T$, we deduce that $\sup \left\{\left\langle a^{*}, w-a\right\rangle: a^{*} \in T(a)\right\} \geq 0$ for all $w \in V_{x} \cap C$. Using the convexity of $C$, we get condition (10). Moreover, when $T(a)$ is convex, weak* compact, one has

$$
\inf _{x \in C} \max _{a^{*} \in T(a)}\left\langle a^{*}, x-a\right\rangle \geq 0 .
$$

Now, applying the Sion minimax theorem or [3, Lemma 2.1(iii)], we get

$$
\max _{a^{*} \in T(a)} \inf _{x \in C}\left\{\left\langle a^{*}, x-a\right\rangle\right\} \geq 0 .
$$

Hence there exists some $a^{*} \in T(a) \subset \partial f(a)$ such that $-a^{*} \in N(C, a)$.

Now let us turn to optimality conditions formulated in terms of normal cones to sublevel sets. The following result can be couched in terms of the adjusted normal cone to $f$ as in [5, Prop. 5.1]; here we give a short proof which avoids this concept.

Proposition 17 Let $f$ be a continuous, semi-strictly quasiconvex function on a convex $C$ and let $a \in X$ be such that $f(a)>\inf _{X} f$. Then $a \in S$ if, and only if, $0 \in N_{f}(a) \backslash\{0\}+N(C, a)$.

Proof. Since $a \in S$ we have that $S_{f}^{<}(a)$ is disjoint from $C$. Since these two sets are convex and $S_{f}^{<}(a)$ is open, the Hahn-Banach theorem yields some $a^{*} \in X^{*} \backslash\{0\}$ and some $\alpha \in \mathbb{R}$ such that

$$
\forall w \in S_{f}^{<}(a), \forall x \in C \quad\left\langle a^{*}, w\right\rangle>\alpha \geq\left\langle a^{*}, x\right\rangle .
$$

Since $f$ is semi-strictly quasiconvex, $a$ belongs to $C$ and the closure of $S_{f}^{<}(a)$. Thus $\left\langle a^{*}, a\right\rangle=\alpha$ and

$$
\forall w \in S_{f}^{<}(a), \forall x \in C \quad\left\langle a^{*}, w-a\right\rangle>0 \geq\left\langle a^{*}, x-a\right\rangle .
$$


Therefore $a^{*} \in N(C, a)$ and $-a^{*} \in N_{f}(a) \backslash\{0\}$ since $N_{f}^{<}(a)=N_{f}(a)$ by Lemma 3 .

Now, assume that $0 \in N_{f}(a) \backslash\{0\}+N(C, a)$. By Lemma $3, f$ is $N_{f} \backslash\{0\}$-pseudoconvex at $a$. Hence, we get $a \in S$ by Proposition 12(a).

Under special assumptions, one has a stronger necessary condition.

Proposition 18 (a) If $-f$ is $(-\partial f)$-protoconvex at $a \in S$, then $-\partial f(a) \subset N(C, a)$.

(b) If $-f$ is eventually $(-\partial f)$-protoconvex at $a \in S$, then condition (10) is satisfied.

Proof. (a) Let $a \in S$. Since $-f$ is $-\partial f$-protoconvex at $a$ and, for all $x \in C,-f(x) \leq-f(a)$, for all $a^{*} \in \partial f(a)$ one has $\left\langle-a^{*}, x-a\right\rangle \leq 0$ for all $x \in C$ so that $-a^{*} \in N(C, a)$. Thus, $-\partial f(a) \subset N(C, a)$.

(b) Let $a \in S$. Since $-f$ is eventually $-\partial f$-protoconvex at $a$ and, for all $x \in C,-f(x) \leq-f(a)$, there exists $a^{*} \in \partial f(a)$ such that $\left\langle a^{*}, x-a\right\rangle \geq 0$. Thus, we get $\inf _{x \in C} \max _{a^{*} \in \partial f(a)}\left\langle a^{*}, x-a\right\rangle \geq 0$.

With a similar proof, we have the following proposition.

Proposition 19 Let $a \in C$ be a strict solution of $(\mathcal{C})$ (i.e. for all $x \in C, f(a)<f(x)$ ).

(a) If $-f$ is $(-\partial f)$-quasiconvex at a, then $-\partial f(a) \subset N(C, a)$.

(b) If $-f$ is $(-\partial f)$-pseudoconvex at a, then $-\partial f(a) \subset N(C, a) \backslash\{0\}$.

Example 6 shows that if we take $\partial f:=-\partial^{0}(-f)$ or $\partial f:=-\partial^{\wedge}(-f)$, the pseudoconvexity assumption of the preceding proposition is automatically satisfied.

\section{$5 \quad$ Mathematical programming problems}

Let us consider now the case in which the constraint set $C$ is defined by a finite family of inequalities, so that problem $(\mathcal{C})$ turns into the mathematical programming problem

$$
(\mathcal{M}) \min f(x) \text { subject to } x \in C:=\left\{x \in X: g_{1}(x) \leq 0, \ldots, g_{n}(x) \leq 0\right\},
$$

where $f: W \rightarrow \mathbb{R}_{\infty}, g_{i}: W \rightarrow \mathbb{R}_{\infty}$ and $W$ is a subset of $X$.

Let $a \in C$, and let $I:=\{1, \ldots, n\}, I(a):=\left\{i \in I: g_{i}(a)=0\right\}$.

Let us first present sufficient optimality conditions.

Proposition 20 Let $f$ be $\partial f$-pseudoconvex at $a \in C$ and $g_{i}$ be $\partial g_{i}$-protoconvex at a for $i \in I(a)$. If for all $x \in C$, there exist $a^{*} \in \partial f(a), a_{i}^{*} \in \partial g_{i}(a)(i \in I(a))$ and $\left(y_{i}\right) \in \mathbb{R}_{+}^{I(a)}$ such that $\left\langle a^{*}+\sum_{i \in I(a)} y_{i} a_{i}^{*}, x-a\right\rangle \geq 0$ (in particular, if $a^{*}+\sum_{i \in I(a)} y_{i} a_{i}^{*}=0$ for some $a^{*} \in \partial f(a)$ and some $a_{i}^{*} \in \partial g_{i}(a)$ for $\left.i \in I(a)\right)$, then a is a solution to problem $(\mathcal{M})$.

Proof. Suppose on the contrary that there exists some $x \in C$ such that $f(x)<f(a)$. Since $f$ is $\partial f$ pseudoconvex at $a$ and $a^{*} \in \partial f(a)$, we have $\left\langle a^{*}, x-a\right\rangle<0$. Since for $i \in I(a), g_{i}$ is $\partial g_{i}$-protoconvex at $a$ and $a_{i}^{*} \in \partial g_{i}(a)$, we have $\left\langle a_{i}^{*}, x-a\right\rangle \leq 0$. Taking $\left(y_{i}\right) \in \mathbb{R}_{+}^{I(a)}$ as in our assumption, we get a contradiction with the relation

$$
\left\langle a^{*}, x-a\right\rangle+\sum_{i \in I(a)} y_{i}\left\langle a_{i}^{*}, x-a\right\rangle<0
$$

deduced from the preceding inequalities.

Remark (a) According to Proposition 4, the condition that $g_{i}$ is $\partial g_{i}$-protoconvex at $a$ can be replaced by quasiconvexity of $g_{i}$ and $\partial g_{i}(a) \subseteq \partial^{r} g_{i}(a)$ for $i \in I(a)$.

(b) By Lemma 2 (b) and (c), the preceding proposition implies [21, Thm. 11].

A variant with a similar proof can be given.

Proposition 21 Let $f$ be eventually $\partial f$-pseudoconvex at $a \in C$ and let $g_{i}$ be eventually $\partial g_{i}$-protoconvex at a for $i \in I(a)$. If for all $x \in C, a^{*} \in \partial f(a)$ and $a_{i}^{*} \in \partial g_{i}(a)(i \in I(a))$ there exists $\left(y_{i}\right) \in \mathbb{R}_{+}^{I(a)}$ such that $\left\langle a^{*}+\sum_{i \in I(a)} y_{i} a_{i}^{*}, x-a\right\rangle \geq 0$, then $a$ is a solution to problem $(\mathcal{M})$. 
Another sufficient condition can be given.

Proposition 22 Let $f$ be $\partial f$-quasiconvex and radially upper semicontinuous at $a \in C$ and let $g_{i}$ be quasiconvex for $i \in I$. Assume that $0 \notin \partial f(a), C^{\perp}=\{0\}$ and $\partial g_{i}(a) \subseteq \partial^{r} g_{i}(a)$ for $i \in I(a)$. If there exists $\left(y_{i}\right) \in \mathbb{R}_{+}^{I(a)}$ such that $a^{*}+\sum_{i \in I(a)} y_{i} a_{i}^{*}=0$ for some $a^{*} \in \partial f(a)$ and some $a_{i}^{*} \in \partial g_{i}(a)$ ( $\left.i \in I(a)\right)$, then $a$ is a solution to problem $(\mathcal{M})$.

Proof. By Proposition 4 (a), $g_{i}$ is $\partial g_{i}$-protoconvex at $a$. Let $a^{*} \in \partial f(a), a_{i}^{*} \in \partial g_{i}(a)$ and $y_{i} \in \mathbb{R}_{+}(i \in I(a))$ be such that $a^{*}+\sum_{i \in I(a)} y_{i} a_{i}^{*}=0$. Since for all $x \in C, i \in I(a)$, one has $g_{i}(x) \leq g_{i}(a)$, hence $\left\langle a_{i}^{*}, x-a\right\rangle \leq 0$, we obtain $-a^{*}=\sum_{i \in I(a)} y_{i} a_{i}^{*} \subseteq N(C, a)$. Then Proposition 13 yields the conclusion.

Now let us turn to necessary optimality conditions for $(\mathcal{M})$. Let us first consider the case of a single constraint which is quasiconvex.

Proposition 23 Let $\partial f: W \rightrightarrows X^{*}$ and let $f$ be $\partial f$-protoconvex. Let $g_{1}$ be quasiconvex, $a \in S$ and $g_{1}(a)=0$. If assumption $(A)$ holds and if $T(a)$ is convex $w^{*}$-compact, then there exists $y_{1} \in \mathbb{R}_{+}$such that

$$
0 \in \partial f(a)+y_{1} N\left(S_{g_{1}}(a), a\right) .
$$

Proof. One has $0 \in \partial f(a)+N(C, a)$ by Proposition 16. On the other hand, since $g_{1}(a)=0$, we have $C=S_{g_{1}}(a)$ and $N(C, a)=N\left(S_{g_{1}}(a), a\right)$. Setting $y_{1}=1$, we have the conclusion.

Now, let us turn to the general case. We shall use the following lemma in which $\Delta:=\left\{\left(x_{i}\right)_{i \in I(a)}\right.$ : $\left.\forall j, k \in I(a), x_{j}=x_{k}\right\}$ is the diagonal of $X^{I(a)}$. Its proof is a consequence of the rule for computing the subdifferential of a sum of convex functions, applied to the case the functions are the indicator functions of the sets $C_{i}:=g_{i}^{-1}((-\infty, 0])$.

Lemma 24 Assume $g_{i}$ is quasiconvex for all $i \in I, g_{i}$ is u.s.c. at a for all $i \in I \backslash I(a)$ and let $C_{i}:=$ $g_{i}^{-1}((-\infty, 0]), a \in X$. Assume that one of the following two conditions holds:

(a) there exist some $k \in I(a)$ and some $z \in C_{k}$ such that $g_{i}(z)<0$ for each $i \in I(a) \backslash\{k\}$ (Slater condition).

(b) $X$ is complete, $g_{i}$ is l.s.c. for all $i \in I(a)$ and $\mathbb{R}_{+}\left(\Delta-\prod_{i \in I(a)} C_{i}\right)=X^{I(a)}$.

Then

$$
N(C, a)=\sum_{i \in I} \mathbb{R}_{+} N\left(C_{i}, a\right) .
$$

This lemma and Proposition 16 entail the following result.

Proposition 25 Let $\partial f: W \rightrightarrows X^{*}$ and let $f$ be $\partial f$-protoconvex. Suppose $g_{1}, \ldots, g_{n}$ satisfy the assumptions of the preceding lemma, assumption $(A)$ holds and $T(a)$ is convex $w^{*}$-compact. Then there exists $\left(y_{i}\right) \in \mathbb{R}_{+}^{I(a)}$ such that

$$
0 \in \partial f(a)+\sum_{i \in I(a)} y_{i} N\left(S_{g_{i}}(a), a\right) .
$$

Note that this optimality condition can be formulated in terms of Gutiérrez functions to the constraints. Recall from [21] that $f$ is a Gutiérrez function at a if its sublevel set $S_{f}(a)$ is convex and such that

$$
N\left(S_{f}(a), a\right)=\mathbb{R}_{+} \partial^{\leq} f(a) .
$$

By the preceding proposition and [21, Lemma 9], if the constraints $\left(g_{i}\right)_{i \in I(a)}$ are Gutiérrez function at $a$ in the preceding proposition as in the preceding lemma, then there exists $\left(y_{i}\right) \in \mathbb{R}_{+}^{I(a)}$ such that $0 \in \partial f(a)+$ $\sum_{i \in I(a)} y_{i} \partial \leq g_{i}(a)$.

Using representations of normal vectors to sublevels sets such as [7, Theorem 3.3.4], [28, Theorem 4.1], [37, Proposition 5.4] under appropriate assumptions, one can derive from the last proposition necessary conditions in fuzzy or limiting forms. 


\section{References}

[1] D. Aussel, Subdifferential properties of quasiconvex and pseudoconvex functions: Unified approach. J. Optim. Theory Appl. 97, 1 (1998), 29-45.

[2] D. Aussel and A. Daniilidis, Normal cones to sublevel sets: an axiomatic approach. Applications in quasiconvexity and pseudoconvexity, in "Generalized Convexity and Generalized Monotonicity", Proceedings of the 6th International Symposium on generalized convexity/Monotonicity, N. Hadjisavvas et al. (eds), Lecture notes in Economics and Mathematical Systems, 502 (2001), Springer, 88-101.

[3] D. Aussel and N. Hadjisavvas, On quasivariational inequalities, J. Optim. Theory Appl. 121, (2004), 445-450.

[4] D. Aussel and N. Hadjisavvas, Adjusted sublevel sets, normal operator, and quasi-convex programming, SIAM J. Optim. 16, 2 (2005), 358-367.

[5] D. Aussel and J. Ye, Quasiconvex programming with locally starshaped contraints region and applications to quasiconvex MPEC, Optimization, to appear.

[6] J. Borde and J.-P. Crouzeix, Continuity properties of the normal cone to the level sets of a quasiconvex function, J. Optim. Theory Appl. 66 (1990), 415-429.

[7] J.M. Borwein and Q. J. Zhu, Techniques of Variational Analysis, Canadian Math. Soc., Springer, New York (2005).

[8] A. Cambini and L. Martein, Generalized convexity and optimality conditions in scalar and vector optimization, In: Handbook of generalized convexity and generalized monotonicity, N. Hadjisavvas, S. Komlosi and S. Schaible (eds.), Kluwer Academic Publishers (2005), 151-194.

[9] J.P. Crouzeix, Criteria for generalized convexity and generalized monotonicity in the differentiable case, In: Handbook of generalized convexity and generalized monotonicity, N. Hadjisavvas, S. Komlosi and S. Schaible (eds.), Kluwer Academic Publishers (2005), 89-120.

[10] A. Daniilidis, N. Hadjisavvas and J.-E. Martínez-Legaz, An appropriate subdifferential for quasiconvex functions, SIAM J. Optim., Vol. 12 (2001), 407-420.

[11] V.F. Demyanov, Convexification and concavification of a positive homogeneous function by the same family of linear functions, Universita di Pisa, (1994) Report 3, 208,802.

[12] V.F. Demyanov and A.M. Rubinov, Contructive nonsmooth analysis, Verlag Peter Lang, Frankfurt am Main, 1995.

[13] W.E. Diewert, Alternative characterizations of six kinds of quasiconcavity in nondifferentiable case with applications to nonsmooth programming, In: Generalized Concavity in Optimization and Economics, S. Schaible and W.T. Ziemba (eds.), Academic Press, New York, 1981.

[14] V. Jeyakuma and D.T. Luc, Nonsmooth calculus, minimality, and monotonicity of convexificators, J. Optim. Theory Appl. 101, 3 (1999), 599-562.

[15] V. Jeyakuma and D.T. Luc, Approximate Jacobian matrices for nonsmooth continuous map and $C^{1}$ optimization, SIAM J. Control and Optim. 36, 5 (1998), 1815-1832.

[16] A. Hassouni and A. Jaddar, On generalized monotone multifunctions with applications to optimality condition in generalized convex programming, J. Inequal. Pure Appl. Math. 4, 67, 11p., electronic. ??

[17] N. Hadjisavvas, Generalized monotonicity and nonsmooth analysis, In: Handbook of generalized convexity and generalized monotonicity, N. Hadjisavvas, S. Komlosi and S. Schaible (eds.), Kluwer Academic Publishers (2005), 465-500. 
[18] V. Jeyakuma and D.T. Luc, Nonsmooth vector functions and continuous optimization, Springer (book to appear).

[19] S. Komlósi, Generalized monotonicity and generalized convexity, J. Optim. Theory Appl. 84, 2 (1995), 361-376.

[20] S. Komlósi, Generalized convexity and generalized derivatives, In: Handbook of generalized convexity and generalized monotonicity, N. Hadjisavvas, S. Komlosi and S. Schaible (eds.), Kluwer Academic Publishers (2005), 421-464.

[21] N.T.H. Linh and J.-P. Penot, Optimality conditions for quasiconvex programming, SIAM J. Optim, Vol. 17,2 (2006), 500-510.

[22] N.T.H. Linh and J.-P. Penot, Generalized affine maps and generalized convex functions, preprint, University of Pau, July 2006.

[23] N.T.H. Linh and J.-P. Penot, Generalized affine functions and generalized differentials, preprint, University of Pau, October 2006.

[24] D.T. Luc, Generalized convexity in vector optimization, In: Handbook of generalized convexity and generalized monotonicity, N. Hadjisavvas, S. Komlosi and S. Schaible (eds.), Kluwer Academic Publishers (2005), 195-236.

[25] O.L. Mangasarian, Nonlinear programming, McGraw-Hill, New York, (1969).

[26] J.E. Martínez-Legaz, On lower subdifferentiable function, in Trend in Mathematical Optimization, K.H. Hoffmanm at al. (eds), Int. Series Numer. Math. 84, Birkhauser, Basel (1988), 197-232.

[27] P. Michel and J.-P. Penot, A generalized derivative for calm and stable functions. Differ. Integral Equ. 5, 2 (1992), 433-454.

[28] H.V. Ngai and M. Thera, A fuzzy necessary optimality condition for non-lipchitz optimization in Asplund spaces, SIAM J. Optim. 12, 3, 656-668.

[29] J.-P. Penot, Are generalized derivatives useful for generalized convex function. In: Generalized Convexity, Generalized Monotonicity, J.-P.Crouzeix, M. Volle, J.-E. Martinez-Legaz (eds.), Kluwer Academic Publishers (1998), 3-39.

[30] J.-P. Penot, What is quasiconvex analysis?, Optimization, 47 (2000), 35-110.

[31] J.-P. Penot, A variational subdifferential for quasiconvex functions, J. Optim. Theory Appl. 111, 3 (2001), 165-171.

[32] J.-P. Penot, Characterization of solution sets of quasiconvex programs, J. Optim. Theory Appl. 117, 3 (2003), 627-636.

[33] J.-P. Penot, A Lagrangian approach to quasiconvex analysis, J. Optim. Theory Appl. 117, 3 (2003), 637647.

[34] J.-P. Penot, Glimpses upon quasiconvex analysis, ESIAM: Proceedings, to appear.

[35] J.-P. Penot and Sach, P.H, Generalized monotonicity of subdifferentials and generalized convexity, J. Optim. Theory Appl. 94, 1 (1997), 251-262.

[36] J.-P. Penot and M. Volle, Surrogate programming and multipliers in quasiconvex programming, SIAM J. Control Optim., 42 (2003), pp. 1994-2003.

[37] J.-P. Penot and C. Zalinescu, Harmonic sums and duality, J. Convex Anal., 7 (2000), pp. 95-113. 
[38] J.-P. Penot and C. Zalinescu, Element of quasiconvex subdifferential calculus, J. Convex Anal., 7 (2000), pp. 243-269.

[39] J.-P. Penot and Quang, P.H, Generalized convexity of functions and generalized monotonicity of set-valued maps, J. Optim. Theory Appl. 92, 2 (1997), 343-356.

[40] P.H. Sach and J.-P. Penot, Characterizations of generalized convexities via generalized directional derivatives, Numerical Functional Analysis and Optimization 19 (1998), 615-634. 